\title{
Spectroscopic binaries among Hipparcos M giants
}

\section{Binary frequency ${ }^{\star}$}

\author{
A. Frankowski ${ }^{1, \star \star}$, B. Famaey ${ }^{1, \star \star \star}$, S. Van $\mathrm{Eck}^{1, \dagger}$, M. Mayor ${ }^{2}$, S. Udry ${ }^{2}$, and A. Jorissen ${ }^{1}$ \\ ${ }^{1}$ Institut d'Astronomie et d'Astrophysique, Université libre de Bruxelles, Faculté des Sciences, CP 226, Boulevard du Triomphe, \\ 1050 Bruxelles, Belgium \\ e-mail: alain.jorissen@ulb.ac.be \\ 2 Observatoire de Genève, Université de Genève, 1290 Sauverny, Switzerland
}

Received 29 July 2008 / Accepted 19 December 2008

ABSTRACT

\begin{abstract}
Context. This paper is the second in a series devoted to studying the properties of binaries with $\mathrm{M}$ giant primaries. Aims. The binary frequency of field $\mathrm{M}$ giants is derived and compared with the binary fraction of $\mathrm{K}$ giants.

Methods. Diagrams of the CORAVEL spectroscopic parameter $S b$ (measuring the average line width) vs. radial-velocity standard deviation for our samples were used to define appropriate binarity criteria. These then served to extract the binarity fraction among the M giants. Comparison is made to earlier data on K giant binarity frequency. The $S b$ parameter is discussed in relation to global stellar parameters, and the $S b$ vs. stellar radius relation is used to identify fast rotators.

Results. We find that the spectroscopic binary detection rate among field M giants, in a sample with few velocity measurements $(\sim 2)$, unbiased toward earlier known binaries, is $6.3 \%$. This is less than half of the analogous rate for field $\mathrm{K}$ giants. This difference originates in the greater difficulty of finding binaries among $\mathrm{M}$ giants because of their smaller orbital velocity amplitudes and larger intrinsic jitter and in the different distributions of $\mathrm{K}$ and $\mathrm{M}$ giants in the eccentricity-period diagram. A higher detection rate was obtained in a smaller $\mathrm{M}$ giant sample with more radial velocity measurements per object: $11.1 \%$ confirmed plus $2.7 \%$ possible binaries. The CORAVEL spectroscopic parameter $S b$ was found to correlate better with the stellar radius than with either luminosity or effective temperature separately. Two outliers of the $S b$ vs. stellar radius relation, HD 190658 and HD 219654, have been recognised as fast rotators. The rotation is companion-induced, as both objects turn out to be spectroscopic binaries.
\end{abstract}

Key words. binaries: spectroscopic - stars: late-type - stars: statistics

\section{Introduction}

This paper is the second in our series discussing the spectroscopic-binary content of a sample of $\mathrm{M}$ giants drawn from the Hipparcos Catalogue (ESA 1997), for which CORAVEL radial velocities have been obtained in a systematic way (Udry et al. 1997; Famaey et al. 2005). The main driver behind such an extensive monitoring effort lies of course with the kinematics; indeed, the kinematical properties of the present sample of $\mathrm{M}$ giants have been fully analysed by Famaey et al. (2005). But this large amout of material may also be used to search for binaries, which is discussed in a series of three papers. In Paper I (Famaey et al. 2009), we present the radial-velocity data and orbital elements of newly-discovered spectroscopic binaries, and also discuss the intrinsic variations sometimes causing pseudo-orbital variations. As a follow-up, the present paper presents the first attempt to derive the frequency of spectroscopic binaries in such an extensive sample of $\mathrm{M}$ giants. A side topic of this paper is the identification of fast rotators among $\mathrm{M}$ giants, using the CORAVEL line-width parameter $S b$. This way

\footnotetext{
* Based on observations carried out at the Swiss telescope installed at the Observatoire de Haute Provence (OHP, France), and at the 1.93-m OHP telescope.

$\star \star$ Postdoctoral Researcher, FNRS, Belgium. Currently at Department of Physics, Technion-Israel Institute of Technology, Haifa 32000, Israel.

$\star \star \star$ Postdoctoral Researcher, FNRS, Belgium.

$\dagger$ Research Associate, FNRS, Belgium.
}

of finding binaries is independent of radial velocities. Finally, we investigate the relation between the CORAVEL line-width parameter $S b$ and stellar parameters and conclude that $S b$ is best correlated with the stellar radius. In Paper III (Jorissen et al. 2009), more evolutionary considerations are presented, based on the analysis of the eccentricity-period diagram of our sample of $M$ giants.

\section{Binary frequency among $M$ giants}

The sample used for the present study and its three subsamples are extensively described in Paper I. Sample I consists of 771 stars from the Hipparcos survey, for which at least 2 radial-velocity measurements have been obtained (Udry et al. 1997). Sample II consists of about one-third sample I stars (254 stars), for which at least 4 measurements have been obtained. Sample III consists of 35 sample II stars with $\sigma\left(V_{\mathrm{r}}\right)>$ $1 \mathrm{~km} \mathrm{~s}^{-1}$, and sample IV of 138 sample II stars with $\sigma\left(V_{\mathrm{r}}\right)<$ $1 \mathrm{~km} \mathrm{~s}^{-1}$.

\subsection{Spectroscopic binaries (sample I)}

As explained in Paper I, the detection of spectroscopic binaries (SBs) among $\mathrm{M}$ giants cannot rely solely on a $\chi^{2}$ test comparing the radial-velocity standard deviation $\sigma(V \mathrm{r})$ to the average instrumental error $\langle\epsilon\rangle$, because of the mass motion existing in the atmospheres of these stars (either due to convection 

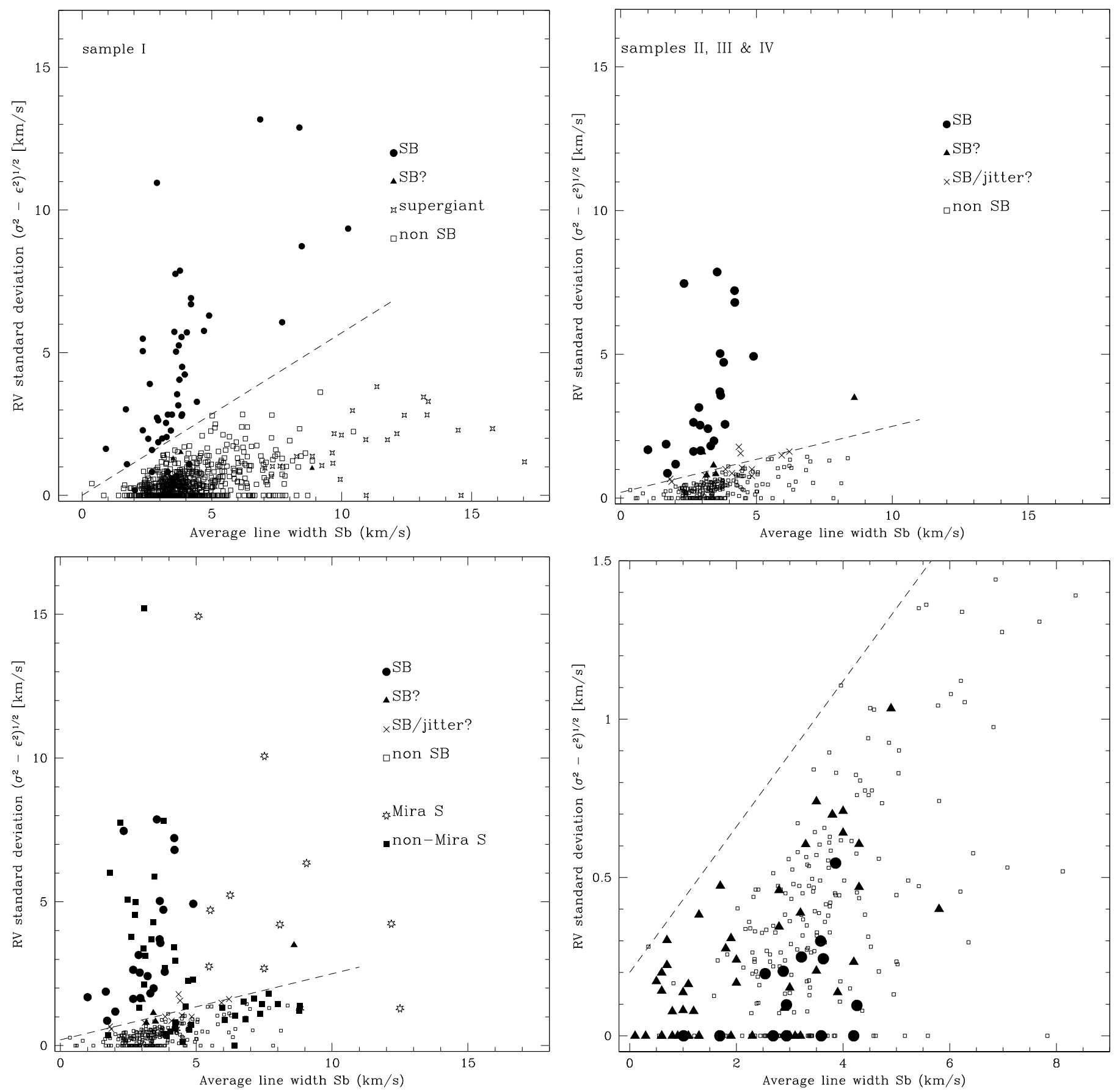

Fig. 1. a) Top left panel: the radial-velocity standard deviation $\sigma_{0}(V \mathrm{r})$ (corrected for the average instrumental error $\langle\epsilon\rangle$ ) as a function of the average intrinsic line width $S b$, for sample I. For non-binary stars (open squares), the maximum radial-velocity jitter increases with $S b$. Filled circles denote binary stars, as identified from their position above the dashed line, combined with the more detailed information gathered from samples II, III, and IV. Diamonds refer to supergiants. b) Top right panel: same as top left panel but for samples II, III and IV. Symbols are as follows: Filled circles: SBs; crosses: SB or jitter; diamond: jitter. The dashed line very clearly marks the limit between SBs (above) and stars with intrinsic radialvelocity jitter (below). c) Bottom left panel: same as b), adding extrinsic S stars from Udry et al. (1998a). Filled triangles: non-Mira S stars; star symbol: Mira S stars. d) Bottom right panel: same as c) after removing the spectroscopic binaries with no orbit and replacing the radial-velocity standard deviation for orbital solutions by the standard deviation of their $\mathrm{O}-\mathrm{C}$ residuals, corrected for the average instrumental error (filled circles). Filled triangles correspond to orbits for barium and extrinsic S stars from Udry et al. (1998a,b). The dashed line and open squares are the same in panels b), c), and d).

or to pulsation), causing intrinsic radial-velocity variations. In Paper I, it was indicated that the parameter $S b$, measuring the average width of spectral lines (corrected for the instrumental width), provides a useful tool to distinguish intrinsic radial-velocity jitter from orbital motion (see Fig. 1$)^{1}$. This is because the $S b$ (i.e., line-width) parameter is a good tracer of the evolutionary state of giant stars (as is shown in Sect. 2.3), and

\footnotetext{
1 Note that the present figure differs slightly from the one published by Famaey et al. (2005), which is in error.
} 
extensive mass motions are occurring in the envelopes of highly evolved giants. These mass motions cause the radial velocity jitter, which is thus large in highly evolved giants, where $S b$ is the largest.

In the $S b-\sigma_{0}$ diagram, where $\sigma_{0}(V \mathrm{r})=\left(\sigma^{2}-\langle\epsilon\rangle^{2}\right)^{1 / 2}$, stars are flagged as binaries if they fall above a "dividing line" (Fig. 1). The binaries identified from the literature and from the more intensive monitoring of subsamples II, III, IV (Sect. 3.1 and Table 4 of Paper I) have been used to define that boundary: the vast majority of these binaries fall above that boundary, while at the same time, confirmed non-binary stars are not found in that region. A dividing line with a slope of 0.57 fulfills these conditions. There is one apparent exception in the lower left corner, HD 40282, which in follow-up observations turned out not to exhibit orbital motion. It may be a statistical fluctuation in the measured jitter. (Compare to the discussion below, where a Gaussian distribution for the radial velocity jitter is assumed). Besides, its status as an exception depends on the exact definitions of $S b$ and of the dividing line. It must be stressed that this slope for sample I is steeper than that obtained for the dividing line in diagrams where the radial-velocity standard deviation is computed from more extended data sets characterising samples II, III, and IV (see the corresponding panels in Fig. 1).

As these lines are defined by the observed distribution of the single-star objects, the difference in the position of the dividing lines can be explained by a combination of two factors: the number of radial-velocity data points for a given object and the total number of objects in a sample.

First, every $\sigma_{0}$ value is an observational estimate of the true standard deviation $\tilde{\sigma}$. It is intuitive that the spread in the values of such an estimator is broader when the number of datapoints (here: radial-velocity measurements) is smaller. Let us assume that the radial-velocity jitter follows a Gaussian distribution, with variance $\tilde{\sigma}^{2}(S b)$ at any given $S b$ value. An estimator of $\tilde{\sigma}^{2}$, the sample variance $s_{N-1}^{2}\left(=\sigma_{0}{ }^{2}\right)$, for an underlying normal distribution follows a scaled $\chi^{2}$ distribution with $N-1$ degrees of freedom, where $N$ is the number of measurements used to compute $s_{N-1}^{2}$. The mean of this $\chi^{2}$ distribution is $\tilde{\sigma}^{2}$ and its variance $2 \tilde{\sigma}^{4} /(N-1)$. The sample standard deviation, $s_{N-1}\left(=\sigma_{0}\right)$, which is an estimator of $\tilde{\sigma}$, in turn follows a scaled $\chi$ distribution with $N-1$ degrees of freedom, an expected value of $c_{4} \tilde{\sigma}$, and a variance of $\left(1-c_{4}^{2}\right) \tilde{\sigma}^{2}$. Here, $c_{4}=\sqrt{2 /(N-1)} \Gamma(N / 2) / \Gamma((N-1) / 2)$ and $\Gamma(x)$ is the gamma function. Therefore the standard deviation of the variable $s_{N-1}$ for $N=2$ (as in sample I) is $\approx 1.55$ times more than for $N=4$ (a value prevailing for samples II, III, and IV). However, the effect of this for the upward spread of $s_{N-1}$ is partially offset by the expected value of $s_{N-1}$ being lower for $N=2$ compared to $N=4$, by a factor of $\sqrt{3} / 2$.

The other contributing factor is that sample I contains about 3 times more objects than sample II. The numbers of (apparently) single objects differ similarly (728 vs. 227 using results from this section - see below - and from Sect. 2.4), which makes the low-probability, large-deviation tail of the $\sigma_{0}$ distribution more populated in sample I. At some value $s_{N-1 \text {,crit }}$, the probabil-

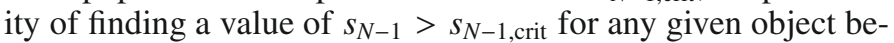
comes less than $1 /$ (sample size). Finding such high $s_{N-1}$ is intuitively "unlikely" - in fact, for large samples this $1 /$ (sample size) limit is equivalent to a probability of $1 / e \approx 0.37$ that no value of

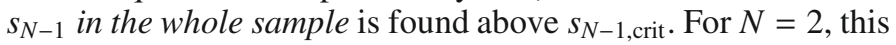
critical value, $s_{N-1, \text { crit }}$, above which the probability $\operatorname{Prob}\left(s_{N-1}\right)$ becomes less than $1 / 728$ is 3.20 . For $\operatorname{Prob}\left(s_{N-1}\right)<1 / 227$, $s_{N-1 \text {,crit }}$ is 2.85 . The ratio of these values is 1.12 , which translates into a ratio of the ranges of $s_{N-1}$ easily populated by the two samples (purely due to their size, as $N=2$ was assumed for both). In fact, these two effects combine: an $N=4$ case with 227 objects (sample II) has $s_{N-1 \text {, crit }}=2.09$, and comparing the ranges of $s_{N-1}$ populated by these samples gives a ratio of 3.20/2.09 $=1.53$. Applying this factor to the dividing line in the $S b-\sigma_{0}$ diagram of sample I, of slope 0.57 , gives an expected slope of 0.372 for sample II. This should be compared to an observed value of $0.3-0.4$, which can be derived for samples II, III, IV when a dividing line going through point $(0,0)$ is adopted, just as for sample I (Fig. 1a). The final detailed position of samples' II, III, IV dividing line is defined in a slightly different way than for sample I, namely from an $S b-\sigma_{0}$ diagram with the orbital motions of known binaries subtracted (Fig. 1d). This adopted line has a positive intercept, resulting in less of a slope: $0.23 S b+0.2$. Nevertheless, the agreement between the above reasoning and the relative positions of the observational dividing lines is evident.

In summary, stars falling above the dividing line may be safely flagged as binaries as long as $S b<5 \mathrm{~km} \mathrm{~s}^{-1}$ (i.e. for non-Mira stars). For higher $S b$ values, the dividing line loses its diagnosis power, since $S b>5 \mathrm{~km} \mathrm{~s}^{-1}$ is the realm of Mira and semi-regular variables which may have intrinsic radial-velocity variations with standard deviations of the order of $10 \mathrm{~km} \mathrm{~s}^{-1}$ (Fig. 1c). For those stars, it is almost impossible to use radial velocities to detect binaries. Methods based on the proper motion may be used instead (see Sect. 2.2).

Using the criterion based on the dividing line, 43 spectroscopic binaries were identified in sample I (upper left panel of Fig. 1), corresponding to a binary frequency of $43 / 771=5.6 \%$. The list of binary stars in sample I may be found in Table A. $1^{2}$ of Famaey et al. (2005).

Because the detection of binaries is difficult among Miras (see Sect. 2.4), we also compute the binary frequency for nonMiras (by limiting sample I to $S b<5 \mathrm{~km} \mathrm{~s}^{-1}$ ), namely 38/603 = $6.3 \%$. That value will be of interest for comparing with $\mathrm{K}$ giants as discussed in Sect. 2.6 and Table 4.

\subsection{Proper-motion binaries}

A few more binary stars have been found from the comparison of the short-term Hipparcos proper motion with the long-term Tycho- 2 proper motion, since only the former is altered by the orbital motion (for orbital periods in the range 1000-30000 d; Frankowski et al. 2007). These "proper-motion-difference binaries" in sample I are listed in Table 1. They are not added, however, to the spectroscopic binary frequency derived in this paper, because the detection efficiency of these binaries involves different selection biases than those associated with the detection of SBs. For instance, proper-motion binaries are efficiently detected only among stars with parallaxes in excess of $\sim 20$ mas, which are rare among $\mathrm{M}$ giants.

\subsection{Fast rotators identified from the CORAVEL line-width parameter $\mathrm{Sb}$}

Another method of finding binaries among $\mathrm{M}$ giants involves identifying fast rotators. Single $M$ giants are not expected to rotate fast (see e.g. De Medeiros et al. 1996; De Medeiros \& Mayor 1999), so that fast rotators can be ascribed to spin-up processes operating in tidally interacting systems (like RS CVn

\footnotetext{
2 The star HIP $26247=\mathrm{RR}$ Cam $=\mathrm{BD}+72^{\circ} 275$ was erroneously flagged as a binary in Famaey et al. (2005), as the result of a confusion between the CORAVEL coding for $\mathrm{BD}+72^{\circ} 275$, and for the binary star $\mathrm{J} 275$ in the Hyades.
} 
Table 1. Supplementary "proper-motion binaries" found in sample I using the method described in Frankowski et al. (2007) and extracted from their Table 2.

\begin{tabular}{|c|c|c|}
\hline $\mathrm{HD} / \mathrm{BD}$ & HIP & Rem. \\
\hline 5006 & 4100 & $\mathrm{SB}^{a}$ \\
\hline +58501 & 12416 & supergiant \\
\hline 51802 & 37391 & \\
\hline 52554 & 33929 & \\
\hline 96572 & 54571 & $\mathrm{SB}^{a}$ \\
\hline 103945 & 58377 & \\
\hline 108907 & 60998 & $\operatorname{SB}^{a}(P=1714 \mathrm{~d})$ \\
\hline 130218 & 72268 & \\
\hline 162159 & 87114 & \\
\hline 190658 & 98954 & $\begin{array}{l}\mathrm{SB}^{a}(P=198.7 \mathrm{~d}) \\
+ \text { visual companion at } 2.3^{\prime \prime}\end{array}$ \\
\hline +394208 & 101023 & supergiant \\
\hline 199871 & 103528 & $\mathrm{SB}^{a}(P=840 \mathrm{~d})$ \\
\hline 209026 & 108588 & \\
\hline 219981 & 115191 & \\
\hline 220088 & 115271 & $\operatorname{SB}^{a}(P=2518 \mathrm{~d})$ \\
\hline
\end{tabular}

$a$ The star is also flagged as spectroscopic binary from the radialvelocity data.

among K giants; De Medeiros et al. 2002). This identification is possible by using the CORAVEL parameter $S b$ (measuring the average width of spectral lines corrected for the instrumental width) to search for outliers in the relation between this parameter and the stellar radius, $R$, as shown in Fig. 3. In previous papers, $S b$ was used as a luminosity proxy (Jorissen et al. 1998; Van Eck \& Jorissen 2000, where it was used to distinguish extrinsic (Tc-poor) from intrinsic (Tc-rich) S stars). Now we have found that the tightest correlation is in fact obtained between $S b$ and the stellar radius, rather than between $S b$ and luminosity, $L$, or effective temperature, $T_{\text {eff }}$. It is the $S b$-radius relationship that is therefore the most appropriate for looking for outliers corresponding to fast rotators.

Before embarking on the search for fast rotators, it is useful to first test the relationship between $S b$ and stellar luminosity, since maximum-likelihood distances are available for all the stars of sample I (Famaey et al. 2005). A possible relation to temperature may also be checked using the $\left(V-I_{\mathrm{C}}\right)-T_{\text {eff }}$ calibration of Bessell et al. (1998) with $\left(V-I_{\mathrm{C}}\right)$ from Platais et al. (2003).

Figure 2 presents the Hertzsprung-Russell diagram for sample I (excluding visual binaries, identified by flag 4 in Table A.1 of Famaey et al. 2005), where $T_{\text {eff }}$ has been derived from the above-mentioned calibration, and the luminosity derives from $M_{\text {bol }}=M_{\mathrm{V}}-\left(V-I_{\mathrm{C}}\right)+B C_{I_{\mathrm{C}}}$, with the bolometric correction $B C_{I_{\mathrm{C}}}$ from Bessell et al. (1998) and $M_{\mathrm{V}}$ from Tycho-2 $V_{T 2}$, combined with the maximum-likelihood distance from Famaey et al. (2005). Two sequences are readily apparent: the lower, most populated one involves giant stars, and the upper one, containing only stars with high $S b$ values, involves supergiant stars. All stars flagged as "Y" (young) by Famaey et al. (2005) belong to the upper sequence. The comparison of the evolutionary tracks with the location of the M giants in the HR diagram of Fig. 2 reveals that they have typical masses in the range $1.2-1.7 M_{\odot}$. As far as $S b$ is concerned, it increases monotonously along the sequences; however, the existence of the two sequences makes it possible to check and exclude the correlation of $S b$ with either $T_{\text {eff }}$ or luminosity alone. Instead, $S b$ correlates well with the stellar radius (Fig. 3), which is a combination of $T_{\text {eff }}$ and $L$.

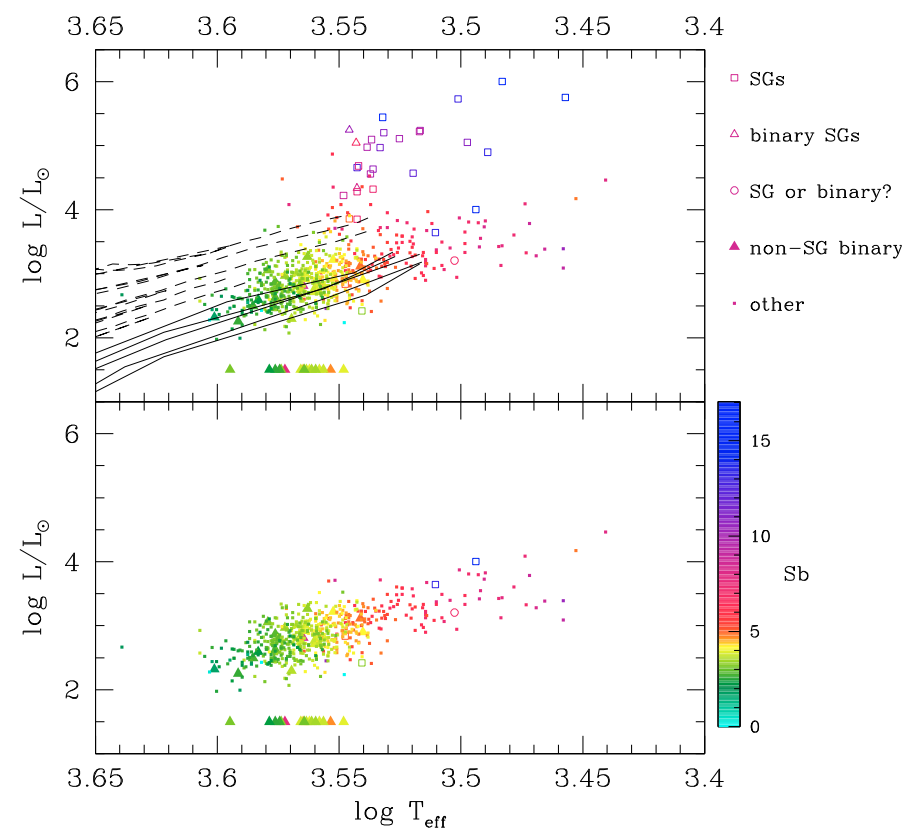

Fig. 2. Upper panel: the HR diagram for the full sample I (but the visual binaries, identified by flag 4 in Table A.1 of Famaey et al. 2005, see text for an explanation about how luminosity and $T_{\text {eff }}$ were derived). The different symbols denote $S b$ ranges as indicated by the labels. Lower panel: same as upper panel after eliminating stars flagged as "Y" (young, supergiant stars) by Famaey et al. (2005). Since maximumlikelihood distances, and hence luminosities, could not be derived by Famaey et al. (2005) for spectroscopic binaries with no orbit, these binaries are displayed in both panels at an arbitrary luminosity of $\log L / L_{\odot}=1.5$. Evolutionary tracks from Charbonnel et al. (1996) (up to the tip of the RGB; no overshooting) for masses $0.9,1.0,1.25,1.5$, and $1.7 M_{\odot}$ are shown as solid lines. Dashed lines are tracks from Schaller et al. (1992) for masses of 2.0, 2.5, 3.0, 4.0, and 5.0 $M_{\odot}$. For low-mass stars, the tip of the RGB corresponds to $S b=5$ to $6 \mathrm{~km} \mathrm{~s}^{-1}$. A colour version of this plot is available in the electronic version only.

There are two stars (HIP8175 = HD 10696 and HIP $98954=\mathrm{HD} 190658)^{3}$ with moderate radii $\left(\log R / R_{\odot}=\right.$ 1.6-1.8) and with $S b$ much larger than expected at the corresponding radii. At larger radii, $\log R / R_{\odot}>2.2$, the relation between $R$ and $S b$ becomes scattered - basically because the CORAVEL cross-correlation dip from which $S b$ is derived become shallow and asymmetric - and the identification of outliers is no longer possible.

HD 219654 is another example of star with too large an $S b$, found in Fig. 2 in the form of a larger square amidst small ones at $\log L / L_{\odot}=1.5$. It does not appear in Fig. 3, because Famaey et al. (2005) did not derive maximum-likelihood distances for that star; therefore, its luminosity and hence radius cannot be derived. The reason for the lack of distance is that the maximumlikelihood distance estimator defined by Famaey et al. (2005) makes use of the radial velocity, and no reliable centre-of-mass velocity could be defined for those stars that appeared to be binaries with rather large velocity amplitude and no orbit available (objects with flag "0" in Famaey et al. 2005).

All fast-rotator candidates are listed in Table 2. Of these, HD 190658 and HD 219654 appear to be genuine fast rotators.

${ }^{3}$ HD 10696 is not displayed on the $R-S b$ plot of Fig. 3, because that star has only one radial-velocity measurement, and was thus removed from sample I. 


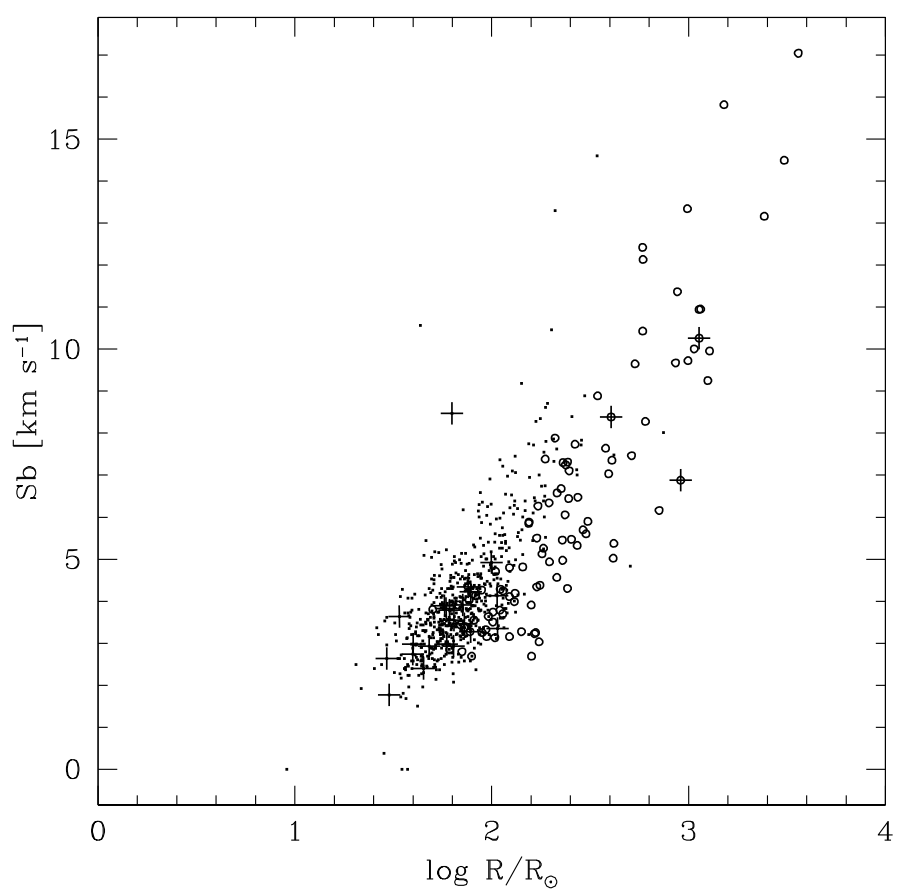

Fig. 3. The relationship between $S b$ and the stellar radius, derived from the Stefan-Boltzmann law and the HR diagram of Fig. 2. Open circles denote stars flagged as "Y" (young) by Famaey et al. (2005) and large crosses identify binaries. The outliers with large $S b$ in this diagram are supposedly binaries, because tidal interactions in the binary spun up or slowed down the giant's rotation. Binaries with no orbits are not included in this figure, since they have no maximum-likelihood distances available either.

The calibration of $S b$ in terms of the rotational velocity $V \sin i$ is from Benz \& Mayor (1981). Fast rotation is expected in binaries close enough for tidal interactions to spin up the giant's rotation (for $\mathrm{K}$ giants, the critical period below which synchronisation takes place is $250 \mathrm{~d}$; see De Medeiros et al. 2002). One clear example of this behaviour is provided by HD 190658, the M-giant binary with the second shortest period known (199 d; Table 8 of Paper I), which exhibits ellipsoidal variations as well (Samus 1997). Its $S b$ of $8.5 \mathrm{~km} \mathrm{~s}^{-1}$ formally corresponds to a rotational velocity of $V \sin i=15 \mathrm{~km} \mathrm{~s}^{-1}$. Correcting for a typical (supposedly turbulent) broadening of $3.5 \mathrm{~km} \mathrm{~s}^{-1}$ for giants of this radius (estimated from Fig. 3) yields $V \sin i=10 \mathrm{~km} \mathrm{~s}^{-1}$. The radius derived from assuming synchronisation is then $R \sin i=39 R_{\odot}$. With $\log T_{\text {eff }}=3.565$ and $\log \left(L / L_{\odot}\right)=2.8$, the radius deduced from Stefan-Boltzmann law is $62.2 R_{\odot}$, which implies an orbit inclined at $i=39^{\circ}$. Rotation slower than synchronous would entail an inclination closer to edge-on.

The radial-velocity data clearly reveals that HD 219654 is a spectroscopic binary (see its location on the $S b-\sigma_{0}(V \mathrm{r})$ diagram in Fig. 1a from the parameters listed in Table 2). An outlying $S b$ value thus appears to be a good way to detect a close binary (with periods up to $\sim 300 \mathrm{~d}$ ). However, there is an important caveat: spurious detections are possible when a close visual companion contaminates the giant spectrum and makes it composite with seemingly broadened lines. HD 10696 (= HIP 8175) is a such case, since it is a close visual binary detected by Hipparcos $(H p=8.54$ and 9.09 with a separation of 0.16 arcsec $)$. It is therefore likely that in this case the large $S b$ comes from the composite nature of the spectrum rather than from rapid rotation of the $\mathrm{M}$ giant star.

\subsection{Spectroscopic binaries (samples II, III, IV)}

The binary frequency derived for sample II, considering the supplementary knowledge gained from the extensive monitoring of the set of stars that had $\sigma(V \mathrm{r})>1 \mathrm{~km} \mathrm{~s}^{-1}$ (corresponding to sample III), and from the $\sigma_{0}(V \mathrm{r})-\sigma(H \mathrm{p})$ diagram (where $\sigma(H \mathrm{p})$ is the standard deviation of the Hipparcos $H p$ magnitude; see Paper I), amounts to $17 / 254$ certain binaries, or $6.7 \%$, with a binomial error of $1.6 \%$. On top of these, 3 stars are possibly binaries, or $1.2 \%$. To detect very long-period binaries among sample II, 138 of the 219 stars not present in sample III (spanning the right-ascension range $0^{\mathrm{h}}-16.5^{\mathrm{h}}$ and not already flagged as SBs) have been re-observed, long after the last measurement of sample II (to make up sample IV). With this additional measurement, 5 binaries (and 2 suspected binaries) have been found (Table 2 of Paper I), adding a fraction of $\frac{5 \times(219 / 138)}{254}=3.1 \%$ to the total frequency. Therefore, the estimated fraction of spectroscopic binaries among $\mathrm{M}$ giants is $6.7+3.1=9.8 \%$, plus $2.4 \%$ of suspected binaries. This frequency is 1.6 times higher than the value obtained for sample I, and this is related to the difference in binarity detection limits between samples (Sect. 2.1).

The above frequency should still be corrected for the selection bias acting against detecting binaries with a Mira component. Exact accounting for binaries among Mira variables is difficult. We argue that it is almost impossible to find spectroscopic binaries among Mira variables (and indeed very few are known; Jorissen 2003). From the period-luminosity-radius relationship for Miras (Wood 1990), it is possible to evaluate the minimum admissible orbital period for a Mira of a given pulsation period in a detached system (assuming fundamental pulsation and masses of 1.0 and $0.75 M_{\odot}$ for the Mira star and its companion, respectively; the conclusions are not very sensitive to these masses, fortunately): it ranges from about $1000 \mathrm{~d}$ for a Mira pulsating with $200 \mathrm{~d}$ period to about $2200 \mathrm{~d}$ for a $600 \mathrm{~d}$ pulsator (see Fig. 9.4 of Jorissen 2003). These orbital periods translate into radial-velocity semi-amplitudes smaller than about $10 \mathrm{~km} \mathrm{~s}^{-1}$. Given that some of these stars have intrinsic radialvelocity jitters of the order of several $\mathrm{km} \mathrm{s}^{-1}$ (Fig. 1 and Alvarez et al. 2001), the difficulty of detecting those spectroscopic binaries using radial velocity techniques immediately becomes clear. An alternative, astrometric method based on the comparison between long-term Tycho-2 proper motions (not altered by possible orbital motions) and short-term Hipparcos proper motions (Wielen et al. 1999, and Sect. 2.2) has not yielded any positive detection (because most Mira stars are quite distant, and thus have parallaxes that are too small for this method to be applied meaningfully; Frankowski et al. 2007).

In the $S b-\sigma_{0}(\mathrm{Vr})$ diagram, Mira variables have $S b>$ $5 \mathrm{~km} \mathrm{~s}^{-1}$, and Fig. 1 reveals that stars with $S b \geq 5 \mathrm{~km} \mathrm{~s}^{-1}$ represent a minor fraction of the total sample (less than $10 \%$, as shown by the solid line in Fig. 4). Therefore, any correction factor will have a limited impact, fortunately. A simple way to estimate the binary frequency among $M$ giants, circumventing the difficulty of detecting spectroscopic binaries involving Mira variables, is to restrict the sample to the 225 stars having $S b<5 \mathrm{~km} \mathrm{~s}^{-1}$, for which the efficiency of binary detection is good. The binary frequency then becomes $0.098 \times 254 / 225=0.111$, to which a fraction of 0.027 of suspected binaries should be added.

Finally, our samples contain a few symbiotic and suspected symbiotic stars: 5 in sample I (EG And, T $\mathrm{CrB}, \mathrm{CH} \mathrm{Cyg}$, V934 Her, and 4 Dra) and 2 in sample II (CH Cyg and 4 Dra). Their hot companions are most likely accreting white dwarfs, so their inclusion in the binary statistics of $\mathrm{M}$ giants is disputable, as in this case they correspond to the second $\mathrm{M}$ giant stage in the 
Table 2. Binaries inferred from the high rotational velocity of the giant primary.

\begin{tabular}{rrlllllllll}
\hline \hline HD & HIP & $\mathrm{SB}^{a}$ & $\begin{array}{l}\sigma_{0} \\
\left(\mathrm{~km} \mathrm{~s}^{-1}\right)\end{array}$ & $\begin{array}{l}S b \\
\left(\mathrm{~km} \mathrm{~s}^{-1}\right)\end{array}$ & $\begin{array}{l}V \sin i \\
\left(\mathrm{~km} \mathrm{~s}^{-1}\right)\end{array}$ & $V-I$ & $\begin{array}{l}T_{\mathrm{eff}} \\
(\mathrm{K})\end{array}$ & $M_{V}$ & $\begin{array}{l}R \\
\left(R_{\odot}\right)\end{array}$ & Rem \\
\hline 10696 & 8175 & - & - & 10.5 & - & 2.3 & 3590 & 0.51 & 43 & $\begin{array}{l}\text { not a fast rotator; } S b \text { contam- } \\
\text { inated by close }(0.16 ”) \text { visual } \\
\text { companion }\end{array}$ \\
190658 & 98954 & $\mathrm{y}$ & 8.7 & 8.5 & 10 & 2.1 & 3670 & -0.67 & 63 & $\begin{array}{l}\text { Ellipsoidal variable, spectro- } \\
\text { scopic binary with } P=199 \mathrm{~d} \\
\text { not listed in Table 4b of Paper I } \\
\text { because it only belongs to sam- } \\
\text { ple I }\end{array}$ \\
\hline
\end{tabular}

${ }^{a}$ This column provides the diagnostics of spectroscopic binarity based on the location of the star in the $S b-\sigma_{0}(V \mathrm{r})$ diagram (Fig. 1); HD 219654: SB: JD 2448 843.634: $5.22 \pm 0.4 \mathrm{~km} \mathrm{~s}^{-1}$, JD $2449676.270: 13.84 \pm 0.3 \mathrm{~km} \mathrm{~s}^{-1}$.

system's evolution. Nevertheless, EG And was flagged as binary by the sample I binarity criterion. If the symbiotic systems are excluded in the evaluation of the binary frequency for sample II, this frequency would decrease slightly to 0.106 . (Only 4 Dra has to be excluded, since $\mathrm{CH}$ Cyg, with $S b=7 \mathrm{~km} \mathrm{~s}^{-1}$, above the $5 \mathrm{~km} \mathrm{~s}^{-1}$ threshold, is already excluded from the above estimate). Readers involved in binary population synthesis should thus note that the binary frequency of 0.111 obtained above corresponds to systems with an $\mathrm{M}$ giant primary and a main sequence or white dwarf companion. There is no guarantee, however, that the frequency of 0.106 (obtained above by excluding symbiotic systems) does correspond to the binary frequency for systems with main-sequence companions alone. It cannot be excluded that $\mathrm{M}$ giants with white dwarf companions hide among nonsymbiotic systems, especially for $\mathrm{M}$ giants at the base of the red giant branch (RGB) where mass loss is weak. This issue will be developed further in Sect. 3 of Paper III.

\subsection{Binaries above $\mathrm{Sb}=5 \mathrm{~km} \mathrm{~s}^{-1}$}

A striking feature of Fig. 1 is the nearly complete absence of stars above the dashed line when $S b \geq 5 \mathrm{~km} \mathrm{~s}^{-1}$ in sample II. Sample I also exhibits an (apparent?) lack of stars above the dashed line beyond $S b=5 \mathrm{~km} \mathrm{~s}^{-1}$. In fact, 4 of the 5 binaries observed above $S b=5 \mathrm{~km} \mathrm{~s}^{-1}$ in sample I (Fig. 1) are VV-Ceplike binaries (Cowley 1969), flagged as belonging to the young ("Y") group by Famaey et al. (2005):

- HD 42474 (WY Gem; $S b=8.4, \sigma_{0}=12.9 \mathrm{~km} \mathrm{~s}^{-1}$ ): a VV Cep type binary, consisting of a M 2 supergiant and an early B type main-sequence star (Leedjaerv 1998);

- HD 190658 (V1472 Aql; $S b=8.5, \sigma_{0}=8.7 \mathrm{~km} \mathrm{~s}^{-1}$ ), an ellipsoidal or eclipsing binary of type M2.5 (Lucke \& Mayor 1982);

- HD 208816 (VV Cep; $S b=10.2, \sigma_{0}=9.3 \mathrm{~km} \mathrm{~s}^{-1}$ );

- BD $+61^{\circ} 08\left(\mathrm{KN} \mathrm{Cas} ; S b=6.9, \sigma_{0}=13.2 \mathrm{~km} \mathrm{~s}^{-1}\right)$ : a VVCep-type binary (Cowley 1969).

The last one, HD 219654 (V349 Peg; $S b=7.7, \sigma_{0}=$ $6.1 \mathrm{~km} \mathrm{~s}^{-1}$ ), is a giant rather than a supergiant star. It is nevertheless located among supergiants because it is a fast rotator, spun up by tidal interactions in a close binary, as discussed in Sect. 2.3.

To evaluate whether or not this absence of binaries above $S b=5 \mathrm{~km} \mathrm{~s}^{-1}$ has any statistical significance, the $H_{0}$ hypothesis that the low $S b$ and high $S b$ regions contain the same fraction of stars above the dashed line has been tested against the alternate hypothesis that the frequencies are different.
The expected number of binaries in the large $S b$ region $\left(\tilde{x_{1}}=\right.$ $p_{3} N_{1}$ ) has been computed from the fraction of binaries in the total sample (denoted $p_{3}$ ) applied to the number of stars in the large $S b$ region $\left(N_{1}\right)$. The corresponding standard deviation on $\tilde{x_{1}}$ (denoted $\sigma_{x 1}$ ) is computed from the expression for a hypergeometric distribution, as applies to the variable $x_{1}$ (the number of binaries in the large $S b$ region), given the total number of stars in the sample, $N_{3}$, the total number of binaries, $p_{3} N_{3}$, and the number of stars in the large $S b$ region, $N_{1}: \sigma_{x 1}=$ $\left[N_{1} p_{3}\left(1-p_{3}\right)\left(N_{3}-N_{1}\right) /\left(N_{3}-1\right)\right]^{1 / 2}$. The significance of the reduced, squared difference $c^{2}=\left[\left(\tilde{x}_{1}-x_{1}\right) / \sigma_{x 1}\right]^{2}$ is computed from the $\chi^{2}$ distribution with one degree of freedom. Table 3 lists the $c^{2}$ values and the corresponding values of the significance level $\alpha=0.5 \operatorname{Prob}_{c^{2}}^{\infty}\left(\chi^{2}\right)$ for different choices of the dividing line. For sample I, the stars flagged "Y" by Famaey et al. (2005) have been discarded and two versions of the dividing line were considered: $\sigma_{0}=3 \mathrm{~km} \mathrm{~s}^{-1}$ with different $S b$ thresholds and $\sigma_{0}=0.57 \mathrm{Sb}$. For sample II, the dividing line was $\sigma_{0}=0.23 S b+0.2$. Table 3 reveals that the significance (denoted $\alpha$ ) of the difference is at best about a few percent.

A Kolmogorov-Smirnov test, based on the cumulative distributions displayed in Fig. 4, has been applied to the more populated sample I and confirms that significance level. With a total number of stars of 606 (after removing the visual binaries and the "Y" group), there are 36 stars above the dividing line (having a slope of 0.57 in the $S b-\sigma_{0}(\mathrm{Vr})$ diagram). The maximum absolute difference between the cumulative distributions of the total number of stars and of the number of binaries, as a function of $S b$, is 0.214 (Fig. 4). This difference, clearly due to the lack of "binaries" with $S b \geq 5 \mathrm{~km} \mathrm{~s}^{-1}$, corresponds to a significance level of $7.5 \%$ for rejecting the null hypothesis that the two distributions are the same.

The statistical significance of this lack of binaries is therefore not very high. Yet, such a scarcity would be easy to understand. First, as noted in Sect. 2.3, Sb is correlated with stellar radius. At larger $R$, for given component masses, the minimum possible orbital period (limited by RLOF) is longer and the maximum (edge-on) amplitude of the orbital radial-velocity variations is smaller. Any inclination would diminish the observed orbital motion even more, while not affecting the intrinsic jitter. Hence, a binary with a more extended giant would tend to hide below the dividing line in the $S b-\sigma_{0}$ diagram, among the intrinsic velocity jitter. This point is illustrated by Fig. 5 .

Next, one should also notice that the RGB tip occurs around $S b=5 \mathrm{~km} \mathrm{~s}^{-1}$ (see Fig. 2), thus accounting for how the number of M giants drops dramatically for $S b \geq 5 \mathrm{~km} \mathrm{~s}^{-1}$, the RGB stars living longer than asymptotic giant branch (AGB) stars. If any, a difference in the binary properties of RGB and AGB stars could 
Table 3. Results of a hypergeometric test checking the $H_{0}$ hypothesis that the frequency $p$ of stars above the dividing line is the same on both sides of the $S b$ threshold value.

\begin{tabular}{|c|c|c|c|c|c|c|c|c|c|}
\hline Case & $S b$ & $\sigma_{0}>3$ & $\sigma_{0} \leq 3$ & $N_{i}$ & $p_{i}=x_{i} / N_{i}$ & $\tilde{x}_{1}$ & $\sigma_{x 1}$ & $c^{2}$ & $\alpha$ \\
\hline \multirow[t]{3}{*}{ Sample I } & $\geq 4.5$ & $x_{1}=5^{a}$ & 154 & 159 & 0.031 & 5.77 & 2.03 & 0.145 & 0.35 \\
\hline & $<4.5$ & $x_{2}=17$ & 430 & 447 & 0.038 & & & & \\
\hline & All & $x_{3}=22$ & 584 & 606 & 0.036 & & & & \\
\hline \multirow[t]{3}{*}{ Sample I } & $\geq 5.0$ & 3 & 104 & 107 & 0.028 & 3.88 & 1.76 & 0.253 & 0.31 \\
\hline & $<5.0$ & 19 & 480 & 499 & 0.038 & & & & \\
\hline & All & 22 & 584 & 606 & 0.036 & & & & \\
\hline \multirow[t]{3}{*}{ Sample I } & $\geq 6.0$ & 3 & 60 & 63 & 0.048 & 2.29 & 1.41 & 0.257 & 0.31 \\
\hline & $<6.0$ & 19 & 524 & 543 & 0.035 & & & & \\
\hline & All & 22 & 584 & 606 & 0.036 & & & & \\
\hline \multirow{4}{*}{ Sample I } & & $\sigma_{0}>0.57 \mathrm{Sb}$ & $\overline{\sigma_{0}} \leq 0.57 \mathrm{Sb}$ & & & & & & \\
\hline & $\geq 5.0$ & 2 & 105 & 107 & 0.019 & 6.36 & 2.22 & 3.85 & 0.025 \\
\hline & $<5.0$ & 34 & 465 & 499 & 0.068 & & & & \\
\hline & All & 36 & 570 & 606 & 0.059 & & & & \\
\hline \multirow{4}{*}{ Sample II } & & $\sigma_{0}>0.23 S b+0.2$ & $\sigma_{0} \leq 0.23 S b+0.2$ & & & & & & \\
\hline & $\geq 5.0$ & 1 & 28 & 29 & 0.034 & 3.43 & 1.64 & 2.189 & 0.069 \\
\hline & $<5.0$ & 29 & 196 & 225 & 0.129 & & & & \\
\hline & All & 30 & 224 & 254 & 0.118 & & & & \\
\hline
\end{tabular}

${ }^{a}$ Subscripts 1, 2, and 3 denote quantities for stars above the dividing line, below it, and the total number, respectively.

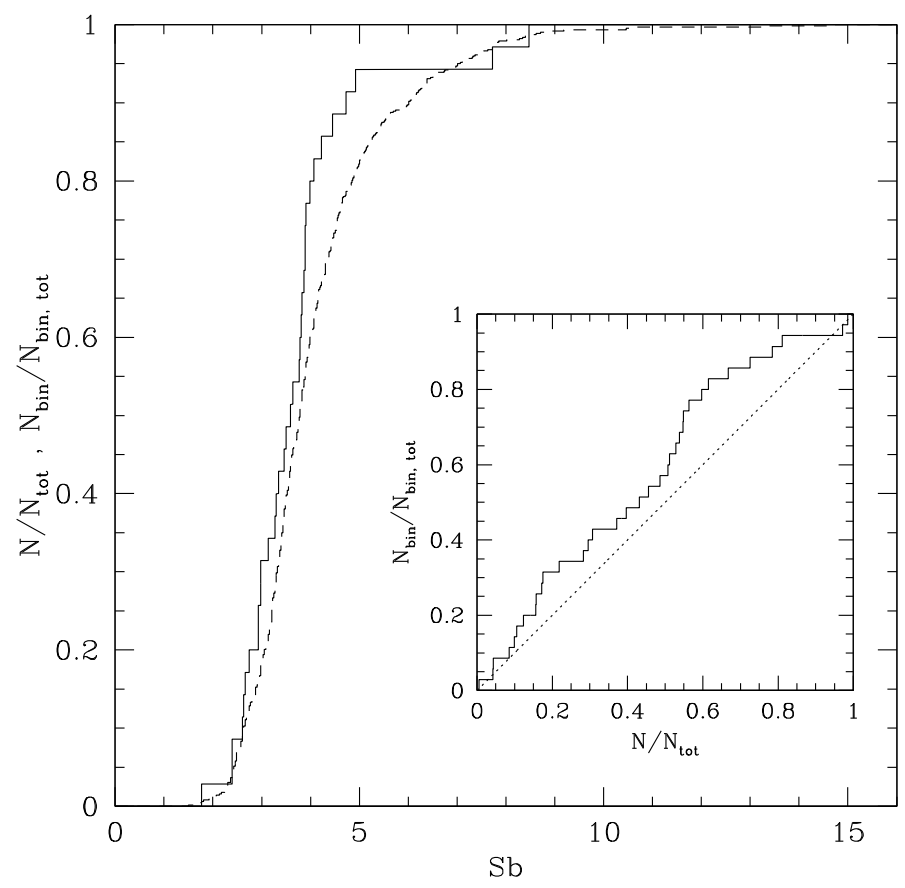

Fig. 4. The cumulative distributions of the total number of stars and of the number of binaries, as a function of $S b$, for sample I (after removing the visual binaries as well as stars flagged "Y" by Famaey et al. 2005). The inset shows how the cumulative distribution of binaries varies throughout the sample. If the frequency of binaries were the same for all values of $S b$, the solid line should follow the diagonal exactly.

involve the large radii reached on the upper RGB: (low-mass) AGB stars would thus be restricted to long-period (i.e., small amplitude) systems, since the shorter-period binaries would have gone through Roche-lobe overflow, with dramatic consequences (dynamical mass transfer, common envelope, and orbital shrinkage to end up as cataclysmic variables) when involving a deep convective envelope, as is the case for RGB stars (and with none of the physical processes to avoid orbital shrinkage, advocated

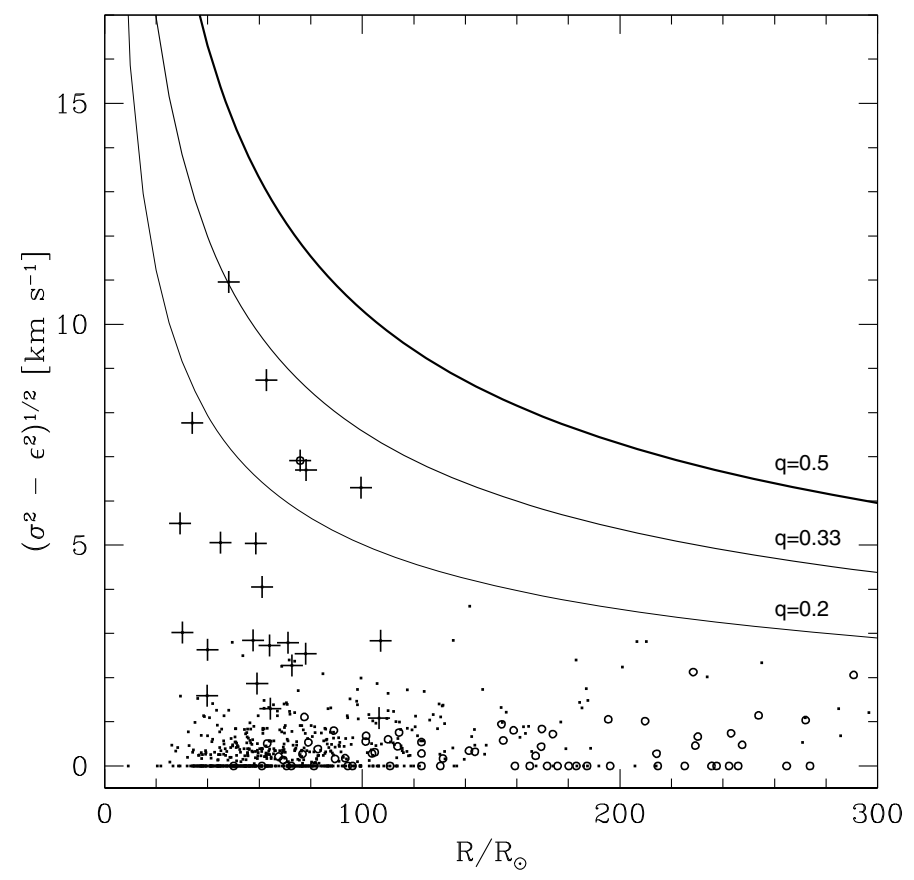

Fig. 5. The radial-velocity jitter versus stellar radius. Circles denote group " $Y$ " stars and crosses binary stars with orbits (binaries with no orbit, i.e., stars flagged "0" in Famaey et al. 2005 are not included as they do not have maximum-likelihood distances available, and hence radius is also not available). The curves correspond to the radial-velocity dispersion that would be exhibited by a giant of $1.5 M_{\odot}$ filling its Roche lobe in an edge-on orbit (with a companion mass fixed by the mass ratio as labelled along the curve). The theoretical radial-velocity semiamplitude has been converted into a standard dispersion by dividing by $\sqrt{2}$.

by Frankowski \& Jorissen 2007 for RLOF involving AGB stars, being operative in RGB stars).

This interpretation is further supported by the period - eccentricity diagram ( $e-\log P$ diagram) presented in Figs. 6 and 7 , revealing that no $\mathrm{M}$ giant is found at orbital periods shorter than $160 \mathrm{~d}$. This limiting period corresponds to a Roche radius of $70 R_{\odot}$ when masses of $1.3 M_{\odot}$ for the giant and $0.6 M_{\odot}$ for the 


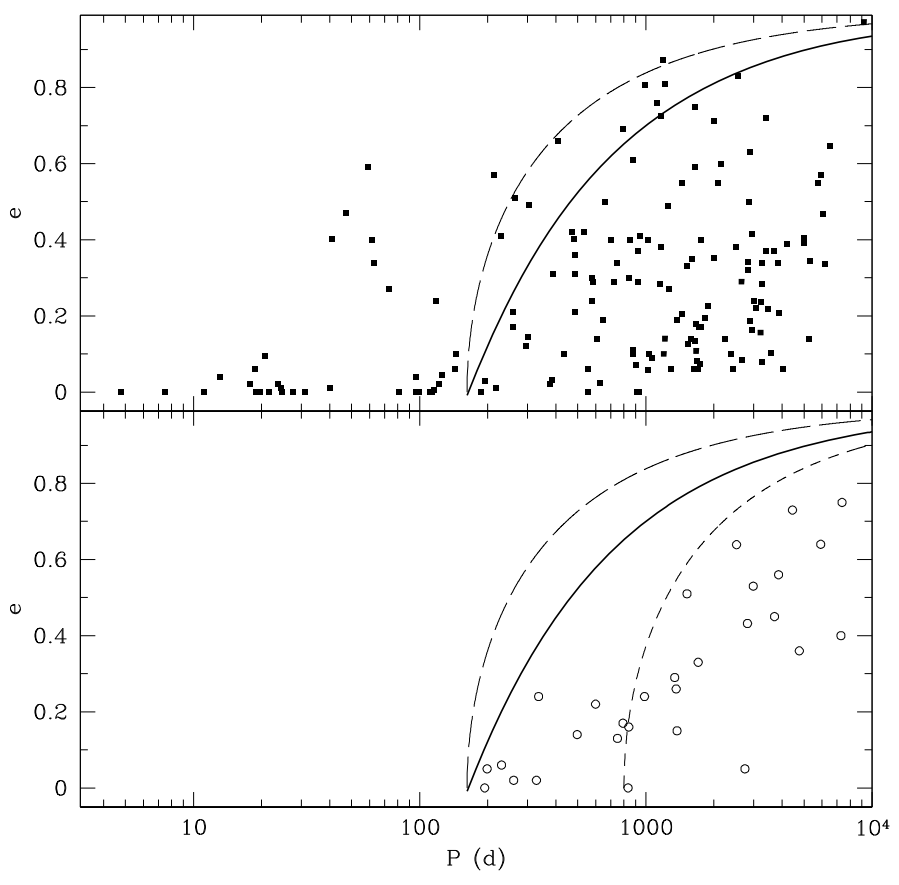

Fig. 6. Top panel: the distribution in the eccentricity-period diagram of the 164 binaries with a KIII primary extracted from the Ninth Catalogue of Spectroscopic Binary Orbits (Pourbaix et al. 2004). The dashed lines correspond to the evolution of the systems during circularisation. The thick solid line represents the locus of constant periastron distance $A(1-e)=157 R_{\odot}$, corresponding to a Roche radius at periastron of $70 R_{\odot}$, assuming masses of 1.3 and $0.6 M_{\odot}$ for the giant and its companion, respectively. Most of the binaries with $P<100 \mathrm{~d}$ are fastrotating, active RS CVn stars. Some of these systems are not yet tidally circularised. They could actually be pre-main-sequence stars (PMS), since RS CVn and PMS stars are sometimes confused (e.g., Rucinski 1977). Star 28 from Torres et al. (2002) is one such case $(P=40.9 \mathrm{~d}$, $e=0.40$, spectral type K3III). Bottom panel: same as top for the M giants. The short-dashed line corresponds to evolution of longer-period systems during circularisation (see Sect. 2.5 for discussion of the lines).

companion are adopted. In fact, no $\mathrm{M}$ giant binary lies to the left of the solid line in Fig. 6, representing the locus of a constant periastron distance $A(1-e)=157 R_{\odot}$ which corresponds to a Roche radius of $70 R_{\odot}$ at periastron for a system with such masses (see Paper III). In contrast, $\mathrm{K}$ giants, which are more compact, may be found at much shorter periods.

However, many $\mathrm{M}$ giants have radii that are much smaller than the $70 R_{\odot}$ threshold obtained above: Fig. 3 shows M giants with radii as small as $30 R_{\odot}$, in agreement with the spread observed by van Belle et al. (1999) for the radii of M giants. Stars with such small radii could in principle be found to the left of the $70 R_{\odot}$ RLOF limit in the $e-\log P$ diagram, but they are not. Part of the explanation could be that the observed envelopes in the $e-\log P$ diagram are defined by tidal interactions more than by RLOF - and tidal interactions operate well before stars fill their Roche lobe. This is especially indicated by the K giants, as they contain a prominent circularised population around and below the minimal period of their $e-\log P$ envelope (Figs. 6, 7). Among the $\mathrm{M}$ giant binaries, the short-period circular subpopulation is less pronounced. Still, they stay within the $70 R_{\odot}$ envelope, not extending down to Roche radii of $\sim 30 R_{\odot}$.

If the $e-\log P$ envelope were due to the tidal interactions, should it not differ in shape from the periastron RLOF type envelope? For comparison, Fig. 6 also displays short- and longdashed lines, corresponding to the evolution of systems during

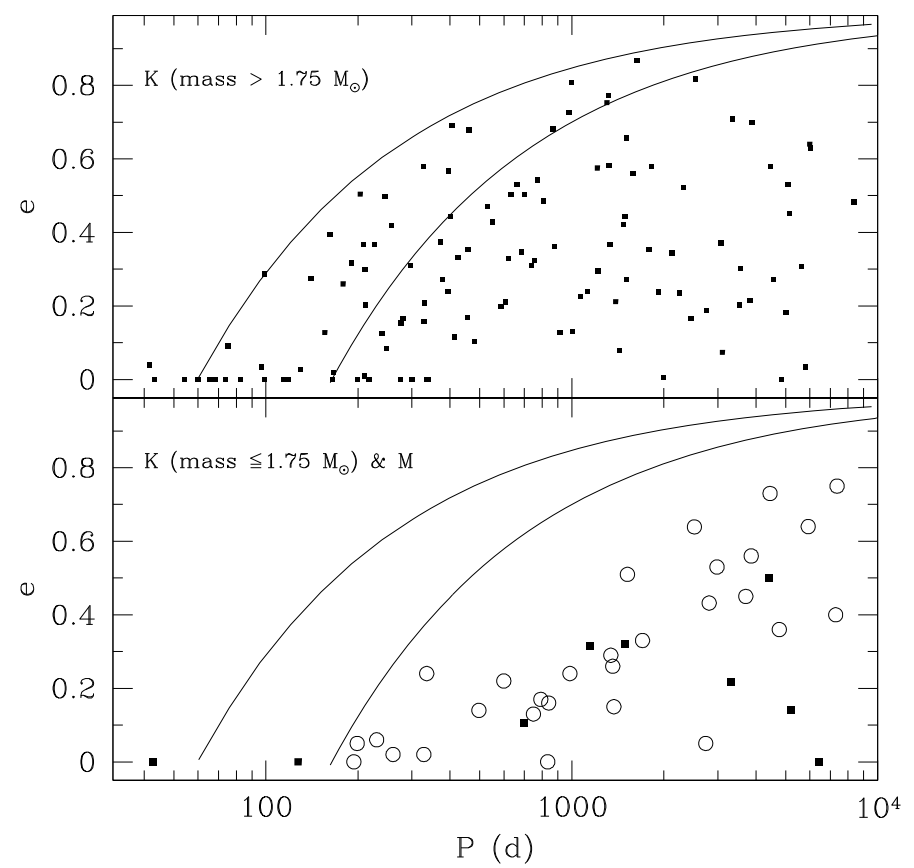

Fig. 7. Top panel: the distribution in the eccentricity-period diagram of $\mathrm{K}$ giants in clusters with a turnoff mass higher than $1.75 M_{\odot}$. Data from Mermilliod et al. (2007). The solid lines represent the loci of constant periastron distances $A(1-e)=79$ and $157 R_{\odot}$, corresponding to a Roche radius at periastron of 35 and $70 R_{\odot}$, respectively. Bottom panel: same as top for the $\mathrm{M}$ giants (open circles) and $\mathrm{K}$ giants (filled squares) in clusters with a turnoff mass lower than or equal to $1.75 M_{\odot}$.

circularisation, which leaves the angular momentum per unit reduced mass $\left[h=A\left(1-e^{2}\right)\right]$ constant (Zahn 1977; Hut 1981). Using Kepler's third law, this condition becomes $P^{2 / 3}(1-$ $\left.e^{2}\right)=$ constant in the eccentricity-period diagram. The two dashed lines in Fig. 6 were plotted adopting the same component masses of $1.3 M_{\odot}$ and $0.6 M_{\odot}$, and with the usual expression for the Roche radius (Eggleton 1983), the Roche radii corresponding to the circular orbits at the base of the two lines in Fig. 6 are 70 and $200 R_{\odot}$. However, lines of this shape do not follow the observed $e-\log P$ envelopes as closely as the lines of constant periastron distance do (especially in the upper panel of Fig. 7). Either the shorter period objects are left outside the circularisation line or a large empty area is included under this supposed envelope. This may be explained as follows. Circularisation becomes fast when the giant comes close to filling its Roche lobe at periastron. But since the circularisation path in the $e-\log P$ diagram is steeper than the periastron line (see Fig. 6), the system evolves somewhat away from the periastron line and thus away from the strong circularisation regime. It does not "slide" all the way down along the circularisation path, because it loses the driver. Only when other effects bring the star and its Roche lobe closer again does circularisation also accelerate again. It should only be fast close to the the periastron envelope. Of these two lines, it is thus the periastron envelope that would regulate the evolution of the $e-\log P$ diagram of an ensemble of systems.

In any case, the minimal Roche lobe radii inferred from the M giant $e-\log P$ diagram are apparently much too large for the measured radii for $\mathrm{M}$ giants. They correspond to Roche lobefilling factors $R / R_{R}$, which are much too small for circularisation to operate efficiently (i.e., on a time scale comparable to the RGB evolutionary time scale). Interestingly enough, a similar difficulty has been pointed out by Mürset \& Schmid (1999) and Mikołajewska (2007) in the context of symbiotic stars. 
Table 4. Summary of the binary frequency among different samples of $\mathrm{K}$ and $\mathrm{M}$ giants.

\begin{tabular}{lllll}
\hline \hline Stellar type & Sample properties & $N_{\text {tot }}$ & Binary frequency (\%) & Ref. \\
\hline Field MIII & I (whatever $N \geq 2$, all $S b)$ & 771 & 5.6 & Sect. 2.1 \\
& II, III, IV $(N>4$, all $S b)$ & 254 & $9.8(+2.4$ ) & Sect. 2.4 \\
Field MIII (without Miras) & I (whatever $N \geq 2, S b<5)$ & 603 & 6.3 & Sect. 2.1 \\
& II, III, IV $(N>4, S b<5)$ & 225 & $11.1(+2.7 ?)$ & Sect. 2.4 \\
Field KIII & (whatever $N \geq 2)$ & $5643^{a}$ & $14.5^{b}$ & Famaey et al. (2005) \\
& Mermilliod \& Mayor (2007) $(N=3)$ & 285 & $16 \pm 2$ & \\
Cluster KIII & Mermilliod \& Mayor (2007) $(N=3)$ & 704 & $30.8 \pm 1.7$ & \\
\hline
\end{tabular}

\footnotetext{
${ }^{a}$ This number differs from the one quoted by Famaey et al. (2005), because we restrict the sample to stars having at least two radial-velocity measurements; ${ }^{b}$ at the level $\operatorname{Prob}\left(\chi^{2}(V \mathrm{r})\right) \leq 0.001$.
}

The former authors have noticed that, surprisingly, the M components in symbiotic stars rarely had Roche lobe-filling factors over 0.6, whereas the latter author notes that symbiotic stars exhibit ellipsoidal variability despite their moderate Roche lobefilling factors. A possible explanation for this discrepancy could be that the usual expression for the Roche radius (Eggleton 1983) overestimates it due to neglecting radiation pressure and other factors countering stellar gravity, which can both shrink the effective Roche-lobe and make surface ellipsoidal distortions important at smaller radii (Schuerman 1972; Frankowski \& Tylenda 2001). This possibility is explored further by Dermine et al. (2009). The observed limit on the giant's Roche-lobe would be explained by a reduction of the effective gravity on the stellar surface to $0.15-0.35$ of its Newtonian value (Frankowski \& Tylenda 2001; Dermine et al. 2009).

From the above discussion one may conclude that the frequency of binaries must be expected to be lower among $\mathrm{M}$ giants than among $\mathrm{K}$ giants, because the larger radii of the former forbid them from being located to the left of the dashed line in the $e-\log P$ diagram of Fig. 6, whereas this restriction does not apply to K giants. This statement will be quantified in Sect. 2.6.

\subsection{Comparison with $\mathrm{K}$ giants}

Not many estimates of the binary frequency among K giants exist in the literature. An early study by Harris \& McClure (1983) of $40 \mathrm{~K}$ giants in the field resulted in a frequency of 15 to $20 \%$ spectroscopic binaries (over a 3 yr time span and with a radial-velocity internal error of $0.40 \mathrm{~km} \mathrm{~s}^{-1}$ ). In open clusters, Mermilliod \& Mayor (2008, in prep.) find a much higher frequency of $30.8 \pm 1.7 \%$ (=217/704) for $\mathrm{G}$ and $\mathrm{K}$ giants under similar observing conditions (similar time span with 3 measurements per star). Surprisingly, for the giants that are not cluster members, the same authors derive a much lower binary frequency, namely $16 \pm 2 \%(=46 / 285)$.

A binary frequency may also be derived for the sample of Hipparcos K giants monitored with CORAVEL and studied by Famaey et al. (2005). Table 4 lists the binary frequency among that sample of $\mathrm{K}$ giants. To compare binary frequencies among $\mathrm{K}$ and $\mathrm{M}$ giants, one should try to avoid systematic and selection effects as much as possible. Most importantly, the comparison should involve binary frequencies derived from the same number of measurements covering approximately the same time span. For $\mathrm{M}$ giants, Table 4 reveals that not respecting this condition may cause the binary frequency estimate to vary by about a factor of two (from $6.3 \%$ in sample I to $11.1 \%$ in samples II, III, IV, after excluding the Miras). Therefore, the binary frequencies among sample I of $\mathrm{M}$ giants and among $\mathrm{K}$ giants were reassessed based on the radial-velocity standard deviations computed only from 2 datapoints (the first and the last available).
The stars flagged as binaries in a given sample may then change. However, it is found that the total number of binaries remains the same (to within 2 units), both for $\mathrm{K}$ and $\mathrm{M}$ giants. Therefore, we may conclude that the fraction of spectroscopic binaries among $\mathrm{M}$ giants (after correcting for the difficulty of finding binaries among Mira variables) is less than among $\mathrm{K}$ giants by a factor $6.3 / 14.5=0.43$.

What could be the origin of such a difference in the binary frequencies among $\mathrm{K}$ and $\mathrm{M}$ giants? Part of it probably comes from the greater difficulty of finding binaries among $M$ giants because of their smaller orbital velocity amplitudes and larger intrinsic jitter (Fig. 5), which prevents a small velocity difference from being ascribed to the orbital motion as it is for $\mathrm{K}$ giants. This is especially true for a small number of measurements, as in our sample I.

Another possibility may reside in the different distributions of $\mathrm{K}$ and $\mathrm{M}$ giants in the eccentricity-period diagram, as shown in Fig. 6. It must be stressed that this figure refers to samples of field giants, i.e., orbits for $\mathrm{K}$ giants being retrieved from the Ninth Catalogue of Spectroscopic Binary Orbits (Pourbaix et al. 2004), explicitely excluding the orbits for $\mathrm{K}$ giants in clusters derived by Mermilliod et al. (2007) (Fig. 7). This allows for a direct comparison of this sample with the sample of $M$ giants without worrying about differences between field and cluster populations.

For stars evolving on the first-ascent giant branch, the radii of $\mathrm{K}$ giants are on average smaller than those of $\mathrm{M}$ giants, so that the minimum orbital period observed for $M$ giants is expected to be somewhat longer than for K giants, as confirmed by Fig. 6. The resulting decrease in binary frequency may be estimated by computing the ratio between the number of $\mathrm{K}$ giants to the right of the solid line to the total number of $\mathrm{K}$ giants, or $118 / 164=0.72$. Applying this factor to the frequency of M giant binaries thus yields $6.3 / 0.72=8.8 \%$, closer to the binary frequency among field $\mathrm{K}$ giants, but still well below it, and this result is robust. For example, if the long-dashed line from Fig. 6 is used as the limit, the correction factor would be $126 / 164=0.77$ instead of 0.72 .

Still another bias, which may alter the factor 0.72 used above, should be taken into consideration. This bias is related to the evolutionary status of the K giant, which may either be the first-ascent giant branch or the core-He burning. For low-mass stars, the latter phase implies that stars have gone through the tip of the RGB where they reached a very large radius (similar to that of $\mathrm{M}$ giants). Therefore, core-He-burning $\mathrm{K}$ giants are expected to have an eccentricity - period distribution similar to that of $\mathrm{M}$ giants.

Thus the eccentricity-period diagram of low-mass $\mathrm{K}$ giants must be expected to mix such $\mathrm{K}$ giants with orbital properties similar to $\mathrm{M}$ giants with $\mathrm{K}$ giants having orbital parameters more 
typical of less evolved (first-ascent giant branch) stars (i.e., short periods and possibly high eccentricities). Since stars spend more time in the He-burning clump, the former should be more numerous, however. For intermediate-mass $\mathrm{K}$ giants, this segregation does not occur since they never went through a stage with large radii. This may be checked by using the sample of $\mathrm{K}$ giant binaries in open clusters (Mermilliod et al. 2007, excluding stars flagged as non-members), where the K-giant mass may be identified with the cluster turnoff mass. Figure 7 reveals that, as expected, the $\mathrm{K}$ giants in clusters with turnoff masses lower than $1.75 M_{\odot}$ are mostly found in the region occupied by $\mathrm{M}$ giants, with only two short-period circular orbits (out of 9), in accordance with the masses of $\mathrm{M}$ giants estimated to fall in the range $1.2-1.7 M_{\odot}$ from Fig. 2 . The situation looks quite different indeed when considering intermediate-mass $\mathrm{K}$ giants (upper panel of Fig. 7). If the sample of $\mathrm{K}$ giant binaries were only made out of low-mass stars, the correcting factor would then be $7 / 9=0.78$ instead of $83 / 126=0.66$ for the intermediate-mass $\mathrm{K}$ giants (close to the value 0.72 derived above for the total sample of field $\mathrm{K}$ giants). The true correction factor should thus be the average of these two values, weighted by the (unknown) fraction of low-mass with respect to intermediate-mass stars among the sample of field K-giant binaries. This true correction factor should be in the range 0.6-0.8 (irrespective of the exact weighing function), which is still too much to account for the factor of 2-3 difference between the binary frequencies among $\mathrm{K}$ and $\mathrm{M}$ giants. In the end, it is the difficulty of finding spectroscopic binaries among $\mathrm{M}$ giants due to their larger radial-velocity jitter that must be invoked to account for the binaries missing among M giants.

\section{Conclusions}

In this paper we have derived the frequency of spectroscopic binaries among field $\mathrm{M}$ giants, for the first time based on an extensive sample, $771 \mathrm{M}$ giants in total. The frequency obtained, $6.3 \%$, is much lower than for $\mathrm{K}$ giants (ranging from 14 to $31 \%$, depending on the samples considered). However, the binary frequency derived from this large sample of $\mathrm{M}$ giants is not very meaningful, since there are important observational difficulties with detecting $\mathrm{M}$ giant binaries, because on average they have smaller orbital velocity amplitudes (longer periods) and larger intrinsic velocity jitter than $\mathrm{K}$ giants. This effect is especially important for samples with only a few measurements per star, as is the case with this sample of $771 \mathrm{M}$ giants.

A higher binary frequency was obtained in a smaller $\mathrm{M}$ giant sample with more radial-velocity measurements per object: $11.1 \%$ confirmed plus $2.7 \%$ possible binaries. Even though this frequency is close to the lower bound of the binary frequency among $\mathrm{K}$ giants, we have shown that they cannot be directly compared because they were obtained under different observing conditions (mostly the number of observations per object). When one tries to compare the binary frequencies for samples of $\mathrm{K}$ and $\mathrm{M}$ giants under similar observing conditions, the binary frequency among $\mathrm{M}$ giants remains lower than that among $\mathrm{K}$ giants by a factor of about 2. Part of this difference may stem from the destruction of shorter-period $\mathrm{K}$ giant binaries due to catastrophic RLOF leading to common envelope, before they can become $\mathrm{M}$ giant binaries. However, how important this effect is depends on the mass distribution in the considered population.

The lack of M-giant binaries with orbital periods shorter than $160 \mathrm{~d}$, as compared to K-giant binaries that have orbital periods as short as $4 \mathrm{~d}$, has been clearly demonstrated from our extensive set of orbital elements.
We have found that the CORAVEL line-width parameter $S b$ is better correlated with the stellar radius than with either luminosity or effective temperature separately. This allowed identification of the $R-S b$ relation outliers HD 190658 and HD 219654 as fast rotators, possibly due to the binary companion influence; indeed, HD 190658 and HD 219654 turn out to be known as spectroscopic binaries. A third candidate was rejected, because its large $S b$ was caused by light contamination from a close visual companion.

Finally, we have shown that $\mathrm{M}$ giants are circularised even though their Roche lobe-filling factor, estimated from the usual expression for the Roche radius, is about 0.5 , in contrast to predictions from tidal circularisation theory. A similar difficulty has been reported for ellipsoidal variables among symbiotic systems. We argue that these discrepancies call for a revision of the Roche-lobe concept in the presence of radiation pressure from the giant component.

Acknowledgements. Stimulating discussions with H. Van Winckel helped to improve this paper. This work was partly funded by an Action de recherche concertée (ARC) from the Direction générale de l'Enseignement non obligatoire et de la Recherche scientifique - Direction de la recherche scientifique Communauté française de Belgique.

\section{References}

Alvarez, R., Jorissen, A., Plez, B., et al. 2001, A\&A, 379, 305 Benz, W., \& Mayor, M. 1981, A\&A, 93, 235 Bessell, M. S., Castelli, F., \& Plez, B. 1998, A\&A, 333, 231

Charbonnel, C., Meynet, G., Maeder, A., \& Schaerer, D. 1996, A\&AS, 115, 339 Cowley, A. P. 1969, PASP, 81, 297

De Medeiros, J. R., \& Mayor, M. 1999, A\&AS, 139, 433

De Medeiros, J. R., Da Rocha, C., \& Mayor, M. 1996, A\&A, 314, 499

De Medeiros, J. R., Da Silva, J. R. P., \& Maia, M. R. G. 2002, ApJ, 578, 943

Dermine, T., Jorissen, A., Siess, L., \& Frankowski, A. 2009, A\&A, submitted Eggleton, P. P. 1983, ApJ, 268, 368

Famaey, B., Jorissen, A., Luri, X., et al. 2005, A\&A, 430, 165

Famaey, B., Pourbaix, D., Frankowski, A., et al. 2009, A\&A, 498, 627 (Paper I)

Frankowski, A., \& Jorissen, A. 2007, Baltic Astron., 16, 104

Frankowski, A., \& Tylenda, R. 2001, A\&A, 367, 513

Frankowski, A., Jancart, S., \& Jorissen, A. 2007, A\&A, 464, 377

Harris, H. C., \& McClure, R. D. 1983, ApJ, 265, L77

Hut P. 1981, A\&A, 99, 126

Jorissen, A. 2003, in Asymptotic Giant Branch Stars, ed. H. Habing, \& H. Olofsson (New York: Springer Verlag), 461

Jorissen, A., Van Eck, S., Mayor, M., \& Udry, S. 1998, A\&A, 332, 877

Jorissen, A., Frankowski, A., Famaey, B., \& Van Eck, S. 2009, A\&A, 498, 489 (Paper III)

Leedjaerv, L. 1998, A\&A, 338, 139

Lucke, P. B., \& Mayor, M. 1982, A\&A, 105, 318

Mermilliod, J.-C., Andersen, J., Latham, D. W., \& Mayor, M. 2007, A\&A, 473, 829

Mikołajewska, J. 2007, Baltic Astron., 16, 1

Mürset, U., \& Schmid, H. M. 1999, A\&AS, 137, 473

Platais, I., Pourbaix, D., Jorissen, A., et al. J. 2003, A\&A, 397, 997

Pourbaix, D., Tokovinin, A. A., Batten, A. H., et al. 2004, A\&A, 424, 727

Rucinski S. M. 1977, PASP, 89, 280

Samus N. N. 1997, Inform. Bull. Variable Stars, 4501, 1

Schaller, G., Schaerer, D., Meynet, G., \& Maeder, A. 1992, A\&AS, 96, 269

Schuerman, D. W. 1972, Ap\&SS, 19, 351

Torres, G., Neuhäuser, R., \& Guenther, E. W. 2002, AJ, 123, 1701

Udry, S., Mayor, M., Andersen, J., et al. 1997, in Hipparcos - Venice, ed. M. Perryman ESA, Noordwijk, ESA SP-402, 693

Udry, S., Jorissen, A., Mayor, M., \& Van Eck, S. 1998a, A\&AS, 131, 25

Udry, S., Mayor, M., Van Eck, S., et al. 1998b, A\&AS, 131, 43

van Belle, G. T., Lane, B. F., Thompson, R. R., et al. 1999, AJ, 117, 521

Van Eck, S., \& Jorissen, A. 2000, A\&A, 360, 196

Wielen, R., Dettbarn, C., Jahreiß, H., Lenhardt, H., \& Schwan, H. 1999, A\&A, 346,675

Wood, P. R. 1990, in From Miras to Planetary Nebulae: Which Path for Stellar Evolution?, ed. M.-O. Mennessier, \& A. Omont (Gif-sur-Yvette: Éditions Frontières), 67

Zahn J.-P. 1977, A\&A, 57, 383 